\title{
SETS OF UNIQUENESS AND SETS OF MULTIPLICITY
}

\author{
BY \\ R. SALEM
}

1.1. Introduction. Let $E$ be a set of points in the interval $(0,2 \pi) . E$ is said to be a set of multiplicity for trigonometrical series if there exists a trigonometrical series not vanishing identically and converging to zero outside $E$. If no such series exists, $E$ is said to be a set of uniqueness. Every set of positive measure is a set of multiplicity, but there exist sets of multiplicity of measure zero. On the other hand, among perfect sets of measure zero there are sets of uniqueness as well as sets of multiplicity.

Let $P$ be a perfect set of measure zero in $(0,2 \pi)$ and let $F(x)$ be any continuous, non-decreasing, purely singular function constructed on $P$, that is to say $F(x)$ is constant in every interval contiguous to $P$, but not everywhere.

Then it is a consequence of Riemann's classical theory of trigonometrical series that if the Fourier-Stieltjes coefficients of $d F$ tend to zero, that is, if

$$
c_{n}=\int_{0}^{2 \pi} e^{n i x} d F=o(1) \quad(n \rightarrow \infty),
$$

$P$ is a set of multiplicity. But if $c_{n} \neq o(1)$ for every $F(x)$ of the above described type constructed on $P$, it is not known whether $P$ is necessarily a set of uniqueness.

The only sets of uniqueness constructed so far are either sets of the "Hardy-Littlewood-Steinhaus type," called sets of the type $H$, or sets which are the sum of a finite number or of a denumerable infinity of $H$-sets, called sets of the type $H_{\sigma}$. For these results, due to Rajchman and to Nina Bary, and for the general theory we refer the reader to Zygmund [1, pp. 291296] ( $\left.{ }^{1}\right)$.

We shall suppose henceforth that $P$ is a perfect set of the Cantor type and of constant ratio of dissection $\xi(0<\xi<1 / 2)$, constructed on $(0,2 \pi)$. Such a set is obtained by dividing the fundamental interval in three parts of lengths proportional to $\xi, 1-2 \xi$, and $\xi$, respectively, and by removing the central open interval. Two intervals are left on which we perform the same operation, and so on indefinitely. (Cantor's classical ternary set corresponds to $\xi=1 / 3$.)

It is easy to see that any point $x$ belonging to $P$ is given by the formula $(1.1 .1) x=2 \pi\left[\epsilon_{1}(1-\xi)+\epsilon_{2} \xi(1-\xi)+\cdots+\epsilon_{k} \xi^{k-1}(1-\xi)+\cdots\right]$ where the $\epsilon_{i}$ are 0 or 1 . Let $F(x)$ be the continuous, non-decreasing, purely

Presented to the Society, February 27, 1943; received by the editors February 2, 1943.

(1) References to bibliography at end of this paper will appear in brackets. 
singular function defined by $F(x)=\epsilon_{1} / 2+\epsilon_{2} / 2^{2}+\cdots+\epsilon_{k} / 2^{k}+\cdots$ when $x$ is given by (1.1.1). Then we have [Carleman 1, p. 225; Salem 1, p. 325]:

$$
c_{n}=\int_{0}^{2 \pi} e^{n i x} d F=e^{\pi n i} \prod_{k=1}^{\infty} \cos \pi n \xi^{k-1}(1-\xi) .
$$

Instead of considering the Fourier-Stieltjes coefficients of $d F$ (depending on the integral parameter $n$ ) we can consider the Fourier-Stieltjes transform of $d F$ (depending on the continuous parameter $v$ ), $F$ being defined to be equal to zero in $(-\infty, 0)$ and to 1 in $(2 \pi, \infty)$. We have thus

$$
c(v)=\int_{-\infty}^{+\infty} e^{v i x} d F=e^{\pi v i} \prod_{k=1}^{\infty} \cos \pi v \xi^{k-1}(1-\xi) .
$$

If the set $P$ and the function $F$ are constructed on the interval $(-\pi /(1-\xi)$, $\pi /(1-\xi))$ instead of the interval $(0,2 \pi)$, we have, denoting by $\Phi$ the function $F((1-\xi) x+\pi)$,

$$
\gamma(u)=\int_{-\infty}^{+\infty} e^{u i x} d \Phi=\prod_{k=0}^{\infty} \cos \pi u \xi^{k} .
$$

We can interpret $\Phi(x)$ as the distribution function which is the convolution of infinitely many symmetric Bernoulli distribution functions:

$$
\Phi(x)=B(x / \pi) * B(x / \pi \xi) * \cdots * B\left(x / \pi \xi^{k}\right) * \cdots,
$$

$B(x)$ being the Bernoulli distribution function equal to zero in $(-\infty,-1)$, to $1 / 2$ in $(-1,+1)$ and to 1 in $(1,+\infty)$. With this interpretation, it is known (see Jessen and Wintner [1]) that $\xi$ need no longer satisfy the condition $0<\xi<1 / 2$, but must satisfy the inequality $0<\xi<1: \gamma(u)$ is still the FourierStieltjes transform of the distribution function $\Phi$, the essential difference being that if $0<\xi<1 / 2$, the "spectrum" of $\Phi$ is the perfect set nowhere dense $P$, and $\Phi$ is purely singular; while if $1 / 2 \leqq \xi<1$ the "spectrum" of $\Phi$ is the whole interval $(-\pi /(1-\xi), \pi /(1-\xi))$, and $\Phi$ can be either purely singular or absolutely continuous.

In what follows, $c_{n}, c(v)$ and $\gamma(u)$ shall always have the meaning of the formulae (1.1.2), (1.1.3) and (1.1.4), respectively.

While, if we are concerned only with the behaviour of $c_{n}$ or of $\gamma(u)$ as $n$ or $u$ tends to $\infty$, we can suppose $0<\xi<1$, it is plain that in the study of perfect sets of uniqueness and of multiplicity we must suppose $0<\xi<1 / 2$.

1.2. It has been known for a long time that the question whether a set $P$ of the above-described type is a set of uniqueness or a set of multiplicity is deeply related to the arithmetical properties of the number $\xi$ (see Nina Bary [2]). Actually it has been shown by Nina Bary [3] and by Kershner [1] that if $\xi$ is rational and equal to the irreducible fraction $p / q, c_{n}$ and $\gamma(u)$ tend to zero if $p \neq 1$, and do not tend to zero if $p=1$. This, as we have seen, implies 
that $P$ is a set of multiplicity when $p \neq 1$, but not necessarily that $P$ is a set of uniqueness when $p=1$. This latter result is, however, true, and has been proved by Nina Bary [3] who has shown that if $p=1$ the set $P$ is of the type $H$, thus solving completely the question whether $P$ is a set of uniqueness or a set of multiplicity when $\xi$ is rational.

The purpose of the present paper is to give the solution of the same problem when $\xi$ is irrational. This solution has been rendered possible due to an interesting work of Pisot concerning the properties of a certain set of algebraic integers which we shall define presently (see Pisot [1] - the same set has also been considered, later on, by Vijayaraghavan [1]). We shall say that $\alpha$ is a "Pisot-Vijayaraghavan number," or briefly a "P. V. number," if $\alpha$ is an algebraic integer such that all its conjugates lie inside the unit circle $|z|<1$. The relation existing between P. V. numbers and the problem of the behaviour of $\gamma(u)$ as $u \rightarrow \infty$ has first been recognized by Erdös [1], who proved that if $1 / \xi$ is a $\mathrm{P}$. V. number, $\gamma(u)$ does not tend to zero for $u \rightarrow \infty$. (The trivial case $\xi=1 / 2$ is excluded.)

Here we shall prove the following theorems:

THEOREM I. Let $G(x)$ be any non-decreasing function, constant in $(-\infty, 0)$ and in $(2 \pi, \infty)$, and let $d_{n}$ and $d(v)$ be the Fourier-Stieltjes coefficient and the Fourier-Stieltjes transform defined by

$$
d_{n}=\int_{0}^{2 \pi} e^{n i x} d G, \quad d(v)=\int_{-\infty}^{+\infty} e^{v i x} d G .
$$

Then, if $d_{n}=o$ (1) for $n \rightarrow \infty$, we have also $d(v)=o(1)$ for $v \rightarrow \infty$. (The converse proposition is, of course, trivial.)

This theorem was proved first independently by A. Zygmund, whose proof, based on different ideas, will be published elsewhere.

In particular we have: Let $0<\xi<1$. If $c_{n}=o(1)$ for $n=\infty$, then $c(v)=o(1)$ for $v=\infty$, and hence $\gamma(u)=o(1)$ for $u=\infty$. In other words, $\gamma(u)$ and $c_{n}$ both tend or do not tend to zero as $u$ and $n$ increase infinitely.

Theorem II. Let $0<\xi<1$. If $\gamma(u)$ does not tend to zero for $u=\infty$, then $1 / \xi$ is necessarily a $P$. V. number.

This theorem is the converse of the result of Erdös quoted above. Collecting the two results we see that $c_{n}$ and $\gamma(u)$ tend to zero if, and only if, $1 / \xi$ is not a P. V. number.

This proves that if $0<\xi<1 / 2$ and $1 / \xi$ is not a $\mathrm{P}$. V. number, the set $P$ is a set of multiplicity. But it does not prove that if $1 / \xi$ is a $P$. V. number, $0<\xi<1 / 2$, the set $P$ is a set of uniqueness. This result is the object of Theorem III.

TheOREM III. Let $0<\xi<1 / 2$. The set $P$ is a set of uniqueness if (and only if) 
$1 / \xi$ is a $P . V$. number. In this case $P$ is a set of the type $H_{\sigma}$.

When $\xi<1 / 2$ approaches $1 / 2$ the middle interval removed in the construction of the set $P$ has a relative length tending to zero, and the Hausdorff dimensionality of the set, which is $\log 2 /|\log \xi|$, tends to 1 . It is, therefore, of some interest to prove that we can have perfect sets of uniqueness constructed with $\xi=1 /(2+\epsilon), \epsilon$ being positive and arbitrarily small. This is the consequence of the following theorem:

Theorem IV. There are $P$. V. numbers of the form $2+\epsilon, \epsilon$ being positive and arbitrarily small. Hence, there are perfect sets $P$ of uniqueness for which the Hausdorff dimensionality is as close to 1 as we please.

2. Proof of Theorem I. We have $d(v)=\int_{-\infty}^{+\infty} e^{v i x} d G=\int_{0}^{2 \pi} e^{v i x} d G$ and $d_{n}=d(n)$. Suppose that $\lim d(n)=0$. If $d(v)$ does not tend to zero, we can find a sequence of numbers $v_{1}, v_{2}, \cdots, v_{p}, \cdots$ increasing infinitely and such that $\left|d\left(v_{p}\right)\right|>\delta, \delta$ being a positive number. Let $v_{p}=n_{p}+\alpha_{p}, n_{p}$ being the integral part, and $\alpha_{p}$ the fractional part of $v_{p}$. There exists an infinite subsequence $\left\{v_{q}\right\}$ of the sequence $\left\{v_{p}\right\}$ such that $\alpha_{q}$ has a limit $\alpha$ when $q \rightarrow \infty$. We have

hence

$$
d\left(v_{q}\right)=\int_{0}^{2 \pi} e^{n_{q} i x} e^{\alpha_{q} i x} d G
$$

$$
\left|\int_{0}^{2 \pi} e^{n_{q} i x} e^{\alpha i x} d G-d\left(v_{q}\right)\right|<[G(2 \pi)-G(0)] \max _{x}\left|e^{\alpha_{q} i x}-e^{\alpha i x}\right|<\epsilon_{q},
$$

$\epsilon_{q}$ tending to zero as $q \rightarrow \infty$. Hence, for $q$ large enough we would have

$$
\left|\int_{0}^{2 \pi} e^{n_{q} i x} e^{\alpha i x} d G\right|>\delta / 2
$$

But this is impossible for it is known as a consequence of Rajchman's theory of formal multiplication of trigonometrical series that $\int_{0}^{2 \pi} e^{n i x} \cos \alpha x d G$ and $\int_{0}^{2 \pi} e^{n i x} \sin \alpha x d G$ tend to zero for $n=\infty$ if, as is the case, $\int_{0}^{2 \pi} e^{n i x} d G$ tends to zero. (See Rajchman [2, p. 688].) Thus the theorem is proved.

3.1. Proof of Theorem II. Suppose that $0<\xi<1$ and that $\gamma(u)$ $=\prod_{k=0}^{\infty} \cos \pi u \xi^{k}$ does not tend to zero for $u=\infty$. This means that there exists a sequence of numbers $u_{1}, u_{2}, \cdots, u_{s}, \cdots$ increasing infinitely and such that $\left|\gamma\left(u_{s}\right)\right|>a, a$ being a positive number. Let $\theta=1 / \xi(1<\theta<\infty)$ and let $\theta^{n-1}<u_{s} \leqq \theta^{n}$, the integer $n=n_{s}$ being a function of $s$. We can write $u_{s}=\lambda_{s} \theta^{n}$ where $1 / \theta<\lambda_{s} \leqq 1$. Hence $\lambda_{s}$ belongs to the interval $(1 / \theta, 1)$ and we can find an infinite subsequence $\left\{u_{q}\right\}$ of the sequence $\left\{u_{s}\right\}$ such that $\lambda_{q}$ has a limit $\lambda$ $(1 / \theta \leqq \lambda \leqq 1)$ when $q \rightarrow \infty$. Now

$$
\left|\gamma\left(u_{q}\right)\right|=\left|\gamma\left(\lambda_{q} \theta^{n_{q}}\right)\right| \leqq\left|\cos \pi \lambda_{q} \cdot \cos \pi \lambda_{q} \theta \cdot \cos \pi \lambda_{q} \theta^{2} \cdots \cos \pi \lambda_{q} \theta^{n_{q}}\right|
$$


hence

$$
\left(1-\sin ^{2} \pi \lambda_{q}\right)\left(1-\sin ^{2} \pi \lambda_{q} \theta\right) \cdots\left(1-\sin ^{2} \pi \lambda_{q} \theta^{n_{q}}\right) \geqq a^{2}
$$

which implies

$$
\exp \left(-\sin ^{2} \pi \lambda_{q}-\sin ^{2} \pi \lambda_{q} \theta-\cdots-\sin ^{2} \pi \lambda_{q} \theta^{n_{q}}\right) \geqq a^{2},
$$

that is to say

$$
\sin ^{2} \pi \lambda_{q}+\sin ^{2} \pi \lambda_{q} \theta+\cdots+\sin ^{2} \pi \lambda_{q} \theta^{n_{q}} \leqq \log 1 / a^{2} .
$$

Let now $u_{r}$ be a number belonging to the sequence $\left\{u_{q}\right\}$ and let $r>q$. We shall have

$$
\sin ^{2} \pi \lambda_{r}+\sin ^{2} \pi \lambda_{r} \theta+\cdots+\sin ^{2} \pi \lambda_{r} \theta^{n_{r}} \leqq \log 1 / a^{2}
$$

and, as $r>q$, we shall have, a fortiori,

$$
\sin ^{2} \pi \lambda_{r}+\sin ^{2} \pi \lambda_{r} \theta+\cdots+\sin ^{2} \pi \lambda_{r} \theta^{n_{q}} \leqq \log 1 / a^{2} .
$$

Let now $r \rightarrow \infty, q$ being fixed. We shall have, as $\lim \lambda_{r}=\lambda$,

$$
\sin ^{2} \pi \lambda+\sin ^{2} \pi \lambda \theta+\cdots+\sin ^{2} \pi \lambda \theta^{n_{q}} \leqq \log 1 / a^{2} .
$$

Since $q$ is arbitrary, this implies that the series

$$
\sum_{0}^{\infty} \sin ^{2} \pi \lambda \theta^{n}
$$

converges to a sum not greater than $\log 1 / a^{2}$. Hence:

Let $\theta=1 / \xi$. If $\gamma(u)$ does not tend to zero for $u=\infty$, there exists a number $\lambda$ $(1 / \theta \leqq \lambda \leqq 1)$ such that the series $\sum_{0}^{\infty} \sin ^{2} \pi \lambda \theta^{n}$ converges.

Once this result is proved, Theorem II is a direct consequence of the theory of Pisot. Since, however, this theory is unnecessarily general for our particular purpose, we think it better to give here a brief account of Pisot's proof for the convenience of the reader.

3.2. Suppose that the series (3.1.1) converges. Let $w_{n}=\lambda \theta^{n}$ and let $w_{n}=a_{n}+\mu_{n}$ where $a_{n}$ is the integer nearest to $w_{n}$ and $-1 / 2<\mu_{n} \leqq 1 / 2$. Then the sequence $\left\{a_{n}\right\}$ is a recurring sequence, in other words

$$
a_{n+k}+b_{1} a_{n+k-1}+\cdots+b_{k} a_{n}=0, \quad n=1,2,3, \cdots,
$$

where $k$ is a fixed integer and $b_{1}, b_{2}, \cdots, b_{k}$ are fixed rational numbers. It is known that in order to prove this it is sufficient to prove that the determinant

$$
D_{n}=\left|\begin{array}{cccc}
a_{0} & a_{1} & \cdots & a_{n} \\
a_{1} & a_{2} & \cdots & a_{n+1} \\
\cdot & \cdot & \cdots & a \\
a_{n} & a_{n+1} & \cdots & a_{2 n}
\end{array}\right|
$$


is equal to zero for all $n \geqq k . k$ being the smallest integer having this property, $k$ is the minimum order of the recurrence. Here we can write

$$
a_{p+1}-\theta a_{p}=w_{p+1}-\theta w_{p}-\left(\mu_{p+1}-\theta \mu_{p}\right)=\theta \mu_{p}-\mu_{p+1}=\eta_{p}
$$

hence

$$
\begin{aligned}
D_{n} & =\left|\begin{array}{ccccc}
a_{0} & a_{1}-\theta a_{0} & a_{2}-\theta a_{1} & \cdots & a_{n}-\theta a_{n-1} \\
a_{1} & a_{2}-\theta a_{1} & a_{3}-\theta a_{2} & \cdots & a_{n+1}-\theta a_{n} \\
\cdot & \cdot & \cdot & \cdots & \cdot \\
a_{n} & a_{n+1}-\theta a_{n} & a_{n+2}-\theta a_{n+1} & \cdots & a_{2 n}-\theta a_{2 n-1}
\end{array}\right| \\
& =\left|\begin{array}{ccccc}
a_{0} & \eta_{0} & \eta_{1} & \cdots & \eta_{n-1} \\
a_{1} & \eta_{1} & \eta_{2} & \cdots & \eta_{n} \\
\cdot & \cdot & \cdot & \cdots \\
a_{n} & \eta_{n} & \eta_{n+1} & \cdots & \eta_{2 n-1}
\end{array}\right|
\end{aligned}
$$

and, by Hadamard's theorem,

$$
D_{n}^{2} \leqq\left(\sum_{0}^{n} a_{p}^{2}\right)\left(\sum_{0}^{n} \eta_{p}^{2}\right)\left(\sum_{1}^{n+1} \eta_{p}^{2}\right) \cdots\left(\sum_{n-1}^{2 n-1} \eta_{p}^{2}\right) .
$$

Now the convergence of (3.1.1) is equivalent to the convergence of $\sum_{0}^{\infty} \mu_{p}^{2}$. Let $\sum_{h}^{\infty} \mu_{p}^{2}=R_{h}$. We have

$$
\begin{gathered}
\eta_{p}^{2} \leqq\left(\theta^{2}+1\right)\left(\mu_{p}^{2}+\mu_{p+1}^{2}\right), \\
\sum_{h}^{n+h} \eta_{p}^{2} \leqq \sum_{h}^{\infty} \eta_{p}^{2} \leqq\left(\theta^{2}+1\right)\left(R_{h}+R_{h+1}\right) \leqq 2\left(\theta^{2}+1\right) R_{h},
\end{gathered}
$$

hence

$$
D_{n}^{2} \leqq\left(\sum_{0}^{n} a_{p}^{2}\right) 2^{n}\left(\theta^{2}+1\right)^{n} R_{0} R_{1} \cdots R_{n-1} .
$$

Now, as $p \rightarrow \infty, a_{p} \sim w_{p}=\lambda \theta^{p}$, hence

$$
\begin{aligned}
a_{0}^{2}+a_{1}^{2}+\cdots+a_{n}^{2} & \sim \lambda^{2}\left(1+\theta^{2}+\cdots+\theta^{2 n}\right) \\
& =\lambda^{2}\left(\theta^{2 n+2}-1\right) /\left(\theta^{2}-1\right)<C \theta^{2 n},
\end{aligned}
$$

$C$ being a constant depending on $\theta$ and $\lambda$ only. Hence

$$
D_{n}^{2} \leqq C\left[2 \theta^{2}\left(\theta^{2}+1\right)\right]^{n} R_{0} R_{1} \cdots R_{n-1}
$$

as $R_{n} \rightarrow 0$ for $n \rightarrow \infty$, we have $D_{n} \rightarrow 0$ for $n \rightarrow \infty$, and as $D_{n}$ is an integer there exists an integer $k$ such that $D_{k-1} \neq 0$ and $D_{n}=0$ for $n \geqq k$; and the sequence $\left\{a_{n}\right\}$ is a recurring sequence of minimum order $k$. 
This, as is well known, proves that the power series $\sum_{0}^{\infty} a_{n} z^{n}$ represents a rational fraction whose denominator is $1+b_{1} z+\cdots+b_{k} z^{k}$. Thus

$$
\sum_{0}^{\infty} a_{n} z^{n}=\frac{P(z)}{1+b_{1} z+\cdots+b_{k} z^{k}},
$$

$P(z)$ being a polynomial with rational coefficients. Now it follows from a theorem of Fatou [1, pp. 368-370, also Pólya and Szegö, 1, Aufgabe 2, pp. 142 and 357] that if a power series with integral coefficients represents a rational fraction, this fraction can be written in the form $P(z) / Q(z), P$ and $Q$ being polynomials with integral coefficients, and $Q(0)$ being equal to 1 . In other words, in (3.2.1), $k$ being the minimum order of the recurrence, $b_{1}, b_{2}, \cdots, b_{k}$ are integers.

Now

$$
\sum_{0}^{\infty} \mu_{n} z^{n}=\sum_{0}^{\infty} \lambda \theta^{n} z^{n}-\sum_{0}^{\infty} a_{n} z^{n}=\lambda \frac{1}{1-\theta z}-\frac{P(z)}{1+b_{1} z+\cdots+b_{k} z^{k}} .
$$

Since $\sum_{0}^{\infty} \mu_{n}^{2}$ converges, the radius of convergence of $\sum_{0}^{\infty} \mu_{n} z^{n}$ is at least 1 . Hence $1 / \theta$ must be a root of $1+b_{1} z+\cdots+b_{k} z^{k}=0$, and the $k-1$ other roots must have moduli not less than 1 . But owing to the convergence of $\sum_{0}^{\infty} \mu_{n}^{2}$, the function $\sum_{0}^{\infty} \mu_{n} z^{n}$ cannot have a pole on the circle $|z|=1$. Hence the conjugates of $1 / \theta$ have their moduli greater than 1 .

It is now sufficient to consider the equation $z^{k}+b_{1} z^{k-1}+\cdots+b_{k}=0$, to see that $\theta$ is an algebraic integer such that all its conjugates lie inside the unit circle $|z|<1$, that is, $\theta$ is a P. V. number, q.e.d.

(The above argument shows also immediately that $\lambda$ is algebraic, but we do not need this result here.)

3.3. Conversely, if $\theta$ is a $\mathrm{P}$. V. number, there exists a number $\lambda$ such that the series (3.1.1) converges. Actually we can take $\lambda=1$. For if $\sigma_{1}, \sigma_{2}, \cdots, \sigma_{k-1}$ are the conjugates of $\theta$, then $\theta^{n}+\sigma_{1}^{n}+\cdots+\sigma_{k-1}^{n}$ is an integer, and if $\left|\sigma_{i}\right| \leqq \delta$ $<1$ for $i=1,2, \cdots, k-1$, we have

$$
\left|\sigma_{1}^{n}\right|+\cdots+\left|\sigma_{k-1}^{n}\right| \leqq(k-1) \delta^{n}<\delta^{\prime n}
$$

where $\delta^{\prime}<1$, if $n$ is large enough. Hence the series $\sum_{0}^{\infty} \sin ^{2} \pi \theta^{n}$ converges, and it converges like a geometric progression.

This requires (except for the trivial case $\theta=2$ ) that $\gamma(u)$ does not tend to zero for $u \rightarrow \infty$, as has been proved by Erdös [1]. The proof is immediate since the convergence of $\sum_{0}^{\infty} \sin ^{2} \pi \theta^{n}$ requires that the infinite product $\prod_{n=0}^{\infty} \cos ^{2} \pi \theta^{n}$ converges to a number $A \neq 0$, unless one of the elements of the product vanishes. This is impossible since it would require $\theta^{n}=(2 k+1) / 2$ for some positive integers $n$ and $k$, which is in contradiction to the nature of the number $\theta$. Hence for $p$ large enough $\prod_{0}^{p} \cos ^{2} \pi \theta^{n}>A / 2$ and 


$$
\begin{aligned}
\left|\gamma\left(\theta^{p}\right)\right| & \left.=\left|\cos \pi \theta^{p} \cdot \cos \pi \theta^{p-1} \cdots \cos \pi \theta \cdot \cos \pi\right| \cdot \mid \cos \pi / \theta \cos \pi / \theta^{2} \cdots\right) \\
& >(A / 2)^{1 / 2}\left|\cos \pi / \theta \cos \pi / \theta^{2} \cdots\right|
\end{aligned}
$$

and the last infinite product is greater than a positive constant except in the trivial case $\theta=2$. (The case $\theta^{n}=2$ with $n>1$ is incompatible with the nature of $\theta$.)

4. Proof of Theorem III. Let now $\xi$ be such that $0<\xi<1 / 2$ and let $\theta=1 / \xi$. We shall prove that if $\theta$ is a $\mathrm{P}$. V. number the set $P$ defined in 1.1 is a set of the type $H_{\sigma}$, hence a set of uniqueness. If $x$ belongs to $P$, we have by (1.1.1)

$$
x=2 \pi(\theta-1)\left[\epsilon_{1} / \theta+\epsilon_{2} / \theta^{2}+\cdots\right], \quad \quad \epsilon_{i}=0 \text { or } 1 .
$$

Let $G$ be the set of the points $y=x /(\theta-1)$. This set lies in the interval $(0,2 \pi /(\theta-1)<2 \pi)$ and we have $y=2 \pi\left[\epsilon_{1} / \theta+\epsilon_{2} / \theta^{2}+\cdots\right]$.

Let $h$ be a fixed integer, to be chosen later on. Consider the $2^{h}$ possible combinations of the $h$ digits $d_{1} d_{2} \cdots d_{h}$ where $d_{i}=0$ or 1 . Let $C_{1}, C_{2}, \cdots, C_{2^{h}}$ denote these combinations. Writing

$$
y=2 \pi\left[\left(\epsilon_{1} / \theta+\cdots+\epsilon_{h} / \theta^{h}\right)+\left(\epsilon_{h+1} / \theta^{h+1}+\cdots+\epsilon_{2 h} / \theta^{2 h}\right)+\cdots\right]
$$

consider the sequence of the $\epsilon_{i}$ belonging to one group of terms in parentheses. They form one of the $2^{h}$ combinations $C_{1}, C_{2}, \cdots, C_{2^{h}}$.

Denote by $G_{s}, s=1,2, \cdots, 2^{h}$, the subset of $G$ such that if $y$ belongs to $G_{s}$ the combination $C_{s}$ occurs in infinitely many groups of terms in parentheses when $y$ is written in the form (4.1). Then obviously $G=G_{1}+G_{2}+\cdots+G_{2^{h}}$ (some of the $G_{s}$ 's may be empty). The $G_{s}$ 's are not necessarily closed sets. But as $G$ is closed we can write, $G_{s}^{\prime}$ being the derived set of $G_{s}$,

$$
G=\left(G_{1}+G_{1}^{\prime}\right)+\left(G_{2}+G_{2}^{\prime}\right)+\cdots+\left(G_{2^{h}}+G_{2^{h}}^{\prime}\right) .
$$

Now let us consider $G_{s}$ for a fixed $s$. Let $m_{1}, m_{2}, \cdots, m_{n}, \cdots$ be the infinite sequence of the ranks of the groups of terms in parentheses where the combination $C_{s}$ occurs. Let $z$ be any point belonging to $G_{s}$. We have

$$
\begin{aligned}
z \theta^{h m_{n}}= & 2 \pi\left[\epsilon_{h m_{n}}+\epsilon_{h m_{n-1}} \theta+\cdots+\epsilon_{h m_{n-1}(h-1)} \theta^{h-1}\right] \\
& +2 \pi\left[\epsilon_{h\left(m_{n-1}\right)} \theta^{h}+\cdots+\epsilon_{1} \theta^{h m_{n}-1}\right] \\
& +2 \pi\left[\epsilon_{h m_{n+1}} / \theta+\epsilon_{h m_{n+2}} / \theta^{2}+\cdots\right] .
\end{aligned}
$$

We can write

$$
z \theta^{h m_{n}}=\alpha(\dot{s})+\beta(z)+y(z)
$$

where $\alpha(s), \beta(z)$ and $y(z)$ denote the three successive brackets in (4.2). It is essential to observe that while $\beta(z)$ and $y(z)$ depend on the particular point $z$, the number $\alpha(s)$ depends on $s$ only but not on $z$. 
Putting $\theta^{n}=a_{n}+\mu_{n}$ where $a_{n}$ is the integer nearest to $\theta^{n}$ and $-1 / 2<\mu_{n}$ $\leqq 1 / 2$, we have

$$
\begin{aligned}
\beta(z) & =2 \pi\left[\epsilon_{h\left(m_{n-1}\right)} a_{h}+\cdots+\epsilon_{1} a_{h m_{n-1}}\right]+2 \pi\left[\epsilon_{h\left(m_{n-1}\right)} \mu_{h}+\cdots+\epsilon_{1} \mu_{h m_{n-1}}\right] \\
& =2 \pi N(z)+\beta_{1}(z),
\end{aligned}
$$

where $N(z)$ is an integer. On the other hand, $\theta$ being a $\mathrm{P}$. V. number, we have by (3.3.1) ( $h$ being supposed large enough)

$$
\left|\beta_{1}(z)\right|<2 \pi\left[\delta^{\prime h}+\delta^{\prime h+1}+\cdots\right]=2 \pi \delta^{\prime h} /\left(1-\delta^{\prime}\right)
$$

where $\delta^{\prime}$ is a fixed number, depending on $\theta$, and essentially inferior to 1 . We have also

$$
z \theta^{h m_{n}}=z a_{h m_{n}}+z \mu_{h m_{n}}
$$

where

$$
\left|z \mu_{h m_{n}}\right|<2 \pi \delta^{\prime m_{n}}<2 \pi \delta^{\prime h}
$$

Finally, let us remark that $y(z)$ is a point belonging to $G_{s}$, hence to $G$.

Let $l$ be the length of the greatest interval contiguous to $G$, and let us suppose that $h$ has been chosen such that the second members of (4.5) and (4.7) are both less than $l / 8$, say. Then we have by (4.3), (4.4), and (4.6)

$$
z a_{h m_{n}}=2 \pi N(z)+\alpha(s)+y(z)+\beta_{2}(z)
$$

where $\beta_{2}(z)=\beta_{1}(z)-z \mu_{h m_{n}}$, and so $\left|\beta_{2}(z)\right|<l / 4$. This is equivalent to

$$
z a_{h m_{n}} \equiv \alpha(s)+y(z)+\beta_{2}(z)
$$

$(\bmod 2 \pi)$.

Let us denote by $G_{s}^{n}$ the set of points deduced from $G_{s}$ by multiplication by the integer $a_{h m_{n}}$ and reduction modulo $2 \pi$. Apart from the translation $\alpha(s)$ which is independent of $z$, the points of $G_{s}^{n}$ are given by the formula $y(z)+\beta_{2}(z)$. As $\left|\beta_{2}(z)\right|<l / 4$, and as $y(z)$ belongs to $G$ (whose greatest contiguous interval is of length $l$ ) it follows that $G_{s}^{n}$ has a contiguous interval of length not less than $l-2 l / 4=l / 2$, and this is true for all $n$.

Now if we consider, instead of $G_{s}$, the closed set $G_{s}+G_{s}^{\prime}$, the multiplication by $a_{h m_{n}}$ and the reduction modulo $2 \pi$ will give us the closed set $G_{s}^{n}+\left(G_{s}^{n}\right)^{\prime}$ where $\left(G_{s}^{n}\right)^{\prime}$ denotes the derived set of $G_{s}^{n}$. It is obvious that for every $n$, the set $G_{s}^{n}+\left(G_{s}^{n}\right)^{\prime}$ will have also a contiguous interval of length not less than $l / 2$, and this means [Rajchman 1, pp. 403-406] that the set $G_{s}+G_{s}^{\prime}$ is a set of the type $H$, hence a closed set of uniqueness.

Now it is known [Nina Bary 1, p. 79, and Zygmund 1, p. 296] that the sum of closed sets of uniqueness is a set of uniqueness, hence the set $G=\sum_{s=1}^{2^{h}}\left(G_{s}+G_{s}^{\prime}\right)$ is a set of uniqueness : indeed, it is a set of the type $H_{\sigma}$.

Finally, the given set $P$ is homothetic to $G$ and they both lie in $(0,2 \pi)$. Hence, by a theorem of Marcinkiewicz and Zygmund [1], $P$ is a set of unique- 
ness. Moreover, it follows from a theorem of Nina Bary [3, p. 714] that $P$ being homothetic to a set of the type $H_{\sigma}$ is itself of the type $H_{\sigma}$.

This completes the proof of Theorem III.

5. Proof of Theorem IV. In order to prove that there exist P. V. numbers of the form $2+\epsilon, \epsilon$ being positive and arbitrarily small, we shall use a method inspired by the work of Vijayaraghavan $[1$, p. 350]. Consider the equation $f(z)=z^{n}-2 z^{n-1}-1=0, n>2$. As $f(2)=-1$, there is a root greater than 2 . We shall prove that for $|z|=1+\eta$ and for all $\eta>0$ small enough $\left(\eta<\eta_{0}\right)$ we have $\left|z^{n}\right|<\left|2 z^{n-1}+1\right|$. For let $z=\rho e^{i \omega}$. We have $\left|z^{n}\right|^{2}=\rho^{2 n}$ and

$$
\left|2 z^{n-1}+1\right|^{2}=1+4 \rho^{n-1} \cos (n-1) \omega+4 \rho^{2 n-2} \geqq 4 \rho^{2 n-2}-4 \rho^{n-1}+1 \text {, }
$$

hence it is sufficient to prove that

$$
\phi(\rho)=\rho^{2 n}-4 \rho^{2 n-2}+4 \rho^{n-1}-1<0 .
$$

But $\phi(1)=0$ and $(d \phi / d \rho)_{\rho=1}=-2 n+4<0$. Hence there exists an $\eta_{0}$ such that if $\rho=1+\eta\left(0<\eta<\eta_{0}\right)$ we have $\left|z^{n}\right|<\left|2 z^{n-1}+1\right|$. This, by Rouché's theorem, proves that $f(z)=0$ has in the circle $|z|=1+\eta, 0<\eta<\eta_{0}$, the same number of roots as $2 z^{n-1}+1=0$, that is to say $n-1$ roots. Hence, letting $\eta \rightarrow 0$, we see that $f(z)=0$ has $n-1$ roots with moduli not greater than 1 . But it is easy to see that there are no roots of modulus 1 , for that would imply

$$
\left\{\begin{array}{l}
\cos n \omega-2 \cos (n-1) \omega=1, \\
\sin n \omega-2 \sin (n-1) \omega=0 .
\end{array}\right.
$$

Squaring and adding, we get $1-4 \cos \omega+4=1$, that is to say $\omega=0$ which is incompatible with the first of the equations (5.1).

Hence the real root greater than 2 is a $P$. V. number. But

$$
f(2(1+\epsilon))=2^{n}(1+\epsilon)^{n}-2^{n}(1+\epsilon)^{n-1}-1=2^{n} \epsilon(1+\epsilon)^{n-1}-1>2^{n} \epsilon-1,
$$

hence taking $\epsilon=1 / 2^{n}$ we see that $f\left(2\left(1+1 / 2^{n}\right)\right)$ is positive. Hence the P. V. number is included between 2 and $2\left(1+1 / 2^{n}\right)$ and as $n$ is as large as we please, this proves the theorem.

6. Remark. In a previous paper [Salem 2, p. 71] the author has considered symmetrical perfect sets $Q$ of order $d$ ( $d$ being a positive integer) and of constant ratio of dissection, whose points are given by the formula

$x=2 \pi\left[\left(\epsilon_{1} / d\right)(1-\xi)+\left(\epsilon_{2} / d\right) \xi(1-\xi)+\cdots+\left(\epsilon_{k} / d\right) \xi^{k-1}(1-\xi)+\cdots\right]$

where $0<\xi<1 /(d+1)$ and the $\epsilon_{i}$ take all values $0,1, \cdots, d$. (The classical case considered in the present paper corresponds to $d=1$.) It has been proved (loc. cit. pp. 77-79) that if $\xi$ is a rational fraction $p / q$ (irreducible), $Q$ is a set of multiplicity if $p \neq 1$, and a set of uniqueness if $p=1$.

The method used to prove this result, combined with the argument of the present paper, proves that $Q$ is a set of uniqueness if and only if $\theta=1 / \xi$ is a P. V. number $(\theta>d+1)$. 


\section{BIBLIOGRAPHY}

BARY, NiNA

1. Sur l'unicitê du développement trigonométrique, Fund. Math. vol. 9 (1927) pp. 62-118.

2. Sur la nature diophantique du problème d'unicité du développement trigonométrique, C. R. Acad. Sci. Paris vol. 202 (1936) p. 1901.

3. Sur le rôle des lois diophantiques dans le problème d'unicité du développement trigonométrique, Rec. Math. (Mat. Sbornik) N.S. vol. 2 (1937) pp. 699-722.

\section{Carleman, $T$.}

1. Sur les équations intégrales singulières a noyau réel et symétrique, Uppsala University Årsskrift, 1923.

ERDös, P.

1. On a family of symmetric Bernoulli convolutions, Amer. J. Math. vol. 61 (1939) pp. 974976.

Fatou, P.

1. Séries trigonométriques et séries de Taylor, Acta Math. vol. 30 (1936) pp. 335-400.

JESSEN, B. AND WINTNER, A.

1. Distribution functions and the Riemann zeta function, Trans. Amer. Math. Soc. vol. 38 (1935) pp. 48-88.

KERSHNER, R.

1. On singular Fourier-Stieltjes transforms, Amer. J. Math. vol. 58 (1936) pp. 450-452.

MARCINKIEWICZ, J. AND ZyGMUND, A.

Pisot, C.

1. Two theorems on trigonometrical series, Rec. Math. (Mat. Sbornik) N. S. vol. 2 (1937) pp. 733-737.

1. La répartition modulo 1 et les nombres algébriques, Annali di Pisa (2) vol. 7 (1938) pp. 205-248.

Pólya, G. AND SZEGö, G.

1. Aufgaben und Lehrsätze aus der Analysis, Berlin, 1925.

RAJCHMAN, A.

1. Sur la multiplication des séries trigonométriques et sur une classe remarquable d'ensembles fermés, Math. Ann. vol. 95 (1926) pp. 389-408.

2. Une classé de séries trigonométriques qui convergent presque partout vers zero, Math. Ann. vol. 101 (1929) pp. 686-700.

SALEM, R.

1. The absolute convergence of trigonometrical series, Duke Math. J. vol. 8 (1941) pp. 317334.

2. On singular monotonic functions of the Cantor type, Journal of Mathematics and Physics vol. 21 (1942) pp. 69-82.

ViJayaraghavan, $T$.

1. On the fractional parts of the powers of a number, Proc. Cambridge Philos. Soc. vol. 37 (1941) pp. 349-357.

\section{ZyGmund, A.}

1. Trigonometrical series, Warszawa-Lwow, 1935.

HARVARD UNIVERSITY,

Cambridge, Mass.

Massachusetts Institute of Technology,

Cambridge, Mass. 